\title{
The Use of Natalizumab for Treatment of MS: Do the Risks Still Outweigh the Gains?
}

Can J Neurol Sci. 2012; 39: 559-560

As the treatment of Multiple Sclerosis (MS) evolves and we move into an era where there is a tendency to push the current disease modifying medications (interferon-beta and glatiramer acetate) to the back of the cupboard in favour of newer, sexier medications, we need also to be reminded of the goals of therapy. The overall hope of treatment is to stave off disease progression by countering the early inflammatory attacks thought to lead to irreparable damage that accumulates with time and eventually pushes most patients into a progressive course. The natural history of relapsing disease is for relapses eventually to cease, MRI changes to become less evident, but patients obviously deteriorate physically and mentally. Though rarely talked about, the mortality ratio of MS patients is some two to three times higher than their non-MS counterparts. We know now that early treatment with medications such as interferon-beta1b can nearly halve the number of deaths over 21 years ${ }^{1}$. But treatment is not just about improving survival, but also about improving the quality of life of a patient. In that regard, reducing the chances of progression is still important.

Disease modifying agents are judged to be effective today based on their ability to reduce relapses, control MRI lesion development and to slow disease progression as measured by the EDSS. Benefits are offset by side effects leading to intolerance or discontinuation and risks of developing other co-morbidities that are sometimes worse than MS itself. Natalizumab is one such treatment, a highly effective agent at controlling the inflammatory events of relapsing disease, which has been relegated to a second "tier" of treatment owing to its peculiar propensity for developing PML, a devastating CNS viral disease caused by the JC virus that most of the time leaves the host in a state far worse than their MS or worse, kills them. A "risk strategy" was developed in the hope of minimizing the development of PML in natalizumab treated patients ${ }^{2}$ by carefully selecting patients according to the presence of antibodies to JC virus, prior exposure to immunosuppressant use or the most crucial risk factor - use beyond 24 months. With nearly 100,000 nataizumab treated MS patients (according to a Biogen-Idec post-marketing analysis as of July 3, 2012), there were 264 confirmed cases of PML (likely an underestimate since not all cases are reported or diagnosed correctly) and the outcome was that 58 patients $(22 \%)$ had died and the remainder left with significant morbidity in the majority of cases. The peak incidence of PML is still after 24 months of treatment where up to $5.16 / 1,000$ treated patients. Of the 264 patients with PML, the antibody status to the JC virus was known in 81 natalizumabtreated patients, but all were positive at the time they developed PML. However, two patients tested negative just nine months prior to developing PML, one patient converting just two months prior coming down with the disease and only one patient with a history of prior immunosuppressive drug use. The test for antiJCV antibodies is therefore not fool-proof, with a false negative rate that is up to $2.7 \%$ and a similar number is estimated for the spontaneous conversion rate per year from negative to positive, so one needs to factor in that nearly $6 \%$ of the "negative" antiJCV antibody status patients in the proposed "risk strategy" could really be positive. Also not factored into this calculation of risk is the problem associated with the use of natalizumab that is somewhat difficult to predict - namely, the tendency for the disease to worsen upon drug cessation. When stopped because of PML, often it is the severe IRIS-like syndrome that leads to significant morbidity and even mortality, but even in the absence of PML, flaring of the underlying MS has been repeatedly reported $^{3}$.

If the benefit of taking natalizumab for their MS far outweighs the risk of PML for patients, then taking the medication would justify its use. It is difficult to measure benefit, but if one looks at just disease progression, then natalizumab might offer a slight advantage over current disease modifying medications such interferon-beta with a lower number-neededto-treat of 8 vs 13 (Interferon-beta1b) ${ }^{4}$ for being "progressionfree" at two years. The amount of progression that is occurring may well be different depending on where patients were and ended up on the EDSS scale such that the meaning of that progression may well be different between the studies. Nevertheless, one needs to then factor in the risk. Mortality figures over 30 years of natural history suggest numbers around $30 \%$ and recent data from a long-term (21 year) follow-up of patients treated early with interferon-beta $1 b^{1}$ suggests that a mortality rate of $22.1 \%$ can be halved with early treatment. Assuming that all the cases of PML occurred thus far in patients over a five year treatment period (though few such patients actually take natalizumab that long), then if one assumes the same five year incidence over 20 years, then there could be $>2,000$ cases of PML (peak incidence of 5.18/1,000) occurring in over 400,000 treated patients. Given that natalizumab-induced PML has been associated with a mortality of $22 \%$, there could be $>400$ iatrogenic deaths in addition to those occurring by natural history. Citing a benefit of a fivefold increase in disease activity free over placebo for natalizumab may sound like it justifies the risk, but being disease activity-free is dependent on how often patients are actually evaluated or scanned, so with a yearly MRI one would expect that less "activity" is found compared to studies with more frequent scanning or evaluations. Regardless, if we focus on being "progression-free" then really there was only a $12 \%$ gain over two years compared to placebo ${ }^{5}$. It is highly likely that the number of "progression-free" patients will diminish over time, while clearly the number of PML cases will rise with continued natalizumab exposure. Will the increase in 
mortality caused by natalizumab be offset by the modest increase in progression-free patients it may have over other therapies in the long-term?

The risk stratification scheme described is reasonable and is clearly needed if we are to avoid as many PML cases as possible. However, we can avoid all PML cases by simply not using natalizumab. PML is thus far a unique complication of natalizumab therapy for MS and death due to interferon-beta or glatiramer acetate use is rare to non-existent. These agents work in most patients for at least some of the time and when the perception is that they are failing to control the disease the recommendation is to switch therapy or escalate to a different agent perceived to offer advantages ${ }^{6}$ even though there is little evidence to justify their use in patients who have clearly "broken through" their first line therapy. This however, is the very indication for current use of natalizumab in most of the country. But benefits must outweigh the risks for patients and in the long term, the increased morbidity and mortality due to natalizumab cannot be claimed to offset the early slight advantage this agent has over the current therapies; and any gains it does have over other therapies appear to be quickly lost once it is discontinued ${ }^{3}$. For these reasons, it should probably not be considered "first line even for patients with milder disease" as suggested, even if antibody status is negative, as being negative still means up to a $6 \%$ chance per year of being or turning positive. For patients with either aggressive or "breakthrough disease" natalizumab is a reasonable choice, regardless of JCV antibody status, as it is important to gain control of disease as quickly as possible. However, until the proposed risk strategy can be shown to reduce the incidence of PML, natalizumab cannot be viewed as a safe long-term maintenance therapy, regardless of JCV antibody status.

\section{REFERENCES}

1. Goodin DS, Reder AT, Ebers GC, et al. Survival in MS: a randomized cohort study 21 years after the start of the pivotal IFNbeta-1b trial. Neurology. 2012;78(17):1315-22.

2. O'Connor P. Natalizumab risk stratification: role of a two-step antiJCV antibody assay. Can J Neurol Sci. 2012;39(5):670-5.

3. Miravalle A, Jensen R, Kinkel RP. Immune reconstitution inflammatory syndrome in patients with multiple sclerosis following cessation of natalizumab therapy. Arch Neurol. 2011; 68(2):186-91.

4. Goodin DS. Disease-modifying therapy in multiple sclerosis: update and clinical implications. Neurology. 2008;71(24 Suppl 3):S8-13.

5. Havrdova E, Galetta S, Hutchinson M, et al. Effect of natalizumab on clinical and radiological disease activity in multiple sclerosis: a retrospective analysis of the Natalizumab Safety and Efficacy in Relapsing-Remitting Multiple Sclerosis (AFFIRM) study. Lancet Neurol. 2009;8(3):254-60.

6. Freedman MS. Multiple Sclerosis therapeutic strategies: use second line agents as first-line agents when time is of the essence. Neurol Clin Prac. 2011;1(1):66-8.
Mark Freedman

The Ottawa Hospital Ottawa, Ontario, Canada 